\title{
Clinical Research Progress of Acupuncture and Moxibustion in the Treatment of Parkinson's Disease Non-Dyskinesia
}

\author{
Chen Tang ${ }^{1}$, Weixing Feng ${ }^{2, *}$, Feifei Yan ${ }^{2}$ \\ ${ }^{1}$ School of Acupuncture and Tuina, Shaanxi University of Chinese Medicine, Xianyang 712046, China. \\ ${ }^{2}$ Affiliated Hospital of Shaanxi University of Chinese Medicine, Xianyang 712000, China.
}

\begin{abstract}
How to cite this paper: Chen Tang, Weixing Feng, Feifei Yan. (2021) Clinical Research Progress of Acupuncture and Moxibustion in the Treatment of Parkinson's Disease Non-Dyskinesia. International Journal of Clinical and Experimental Medicine Research, 5(3), 310-315.

DOI: 10.26855/ijcemr.2021.07.013
\end{abstract}

Received: April 19, 2021

Accepted: May 18, 2021

Published: June 17, 2021

*Corresponding author: Weixing Feng, Affiliated Hospital of Shaanxi University of Chinese Medicine, Xianyang 712000 , China.

Email: 2395613596@qq.com

\begin{abstract}
Parkinson's disease (PD) is a chronic degenerative disease of the central nervous system (CNS) characterized by motor and non-motor disorders. At present, symptomatic treatment of non-motor disorders is mainly used, but due to the side effects of drugs and the complexity of the disease, drugs acting on a certain symptom will affect other symptoms accordingly, so it is difficult to control this unnecessary negative treatment effect clinically. Therefore, it is necessary to continuously search for safer and more effective treatment programs. In recent years, the application of acupuncture and moxibustion in the treatment of non-motor disorders is increasing gradually, and the feedback of efficacy is better. In this paper, by collecting the relevant clinical literature reports in recent years, some collation and discussion are made in the hope of providing reference for the clinical treatment of this disease.
\end{abstract}

\section{Keywords}

Acupuncture Treatment, Parkinson’s Disease, Non-Motor Disorders, The Research Progress

Parkinson disease (PD) has always been regarded as one of the clinical problems that need to be solved. In recent years, as the medical community has paid close attention to PD, it has been found that it is not only manifested by motor symptoms (MS), but also nonmotor symptoms (NMS) that are more difficult to intervene. NMS appeared earlier than MS, and its interaction aggravated the development of the disease. According to the Lancet Global Burden of Disease statistics, 3 years ago, the number of PD patients had reached more than 8 million, and the incidence of PD among people over 60 years old in China was about 1\%-2\% [1-2]. Non-motor symptoms of PD include neuropsychiatric symptoms, autonomic nervous dysfunction, sleep and wakefulness disorders, and sensory disorders, etc. [3]. Acupuncture and moxibustion in the treatment of Parkinson's disease has also risen to a new level along with people's attention to this disease, especially for non-movement disorders with a better relief effect, and has been gradually recommended as the preferred clinical treatment by medical staff [4].

\section{Neuropsychiatric symptoms}

\subsection{Cognitive dysfunction}

One third of clinical patients have already had mild cognitive impairment (MCI) at the time of PD diagnosis, and there is a $20 \%$ risk of exacerbating PD dementia subsequently [5]. Experiments have proved that the mechanism of this disease is caused by the changes of neurotransmitters such as acetylcholine, dopamine and 
5-hydroxytryptamine in the brain caused by brain tissue atrophy or destruction in the frontal cortex, hippocampus and basal ganglia [6]. Acetylcholinesterase inhibitors donepezil and galantamine are the representatives of therapeutic drugs, but the latest research reports show that there is not enough evidence to treat PD dementia [7]. Cao Liangsong [8] selected Baihui, Sishencong, Xuanzhong, parietal and midline, frontal and midline combined cognitive function training, and significantly improved MMSE and MOCA scores after treatment. Zhang Lijuan [9] treated the disease with electroacupuncture moderate stimulation of Baihui and moxibustion Shenque combined with traditional Chinese medicine, and the quality of life of the patients was significantly improved after treatment. Zhang Xiaoli [10] took Baihui, Sishencong, Shenting and other acupoints together with simple drug treatment, which effectively improved the cognitive score. Ye Jiasheng et al. [11] used Baihui, Sishencong and Xuanzhong (Shuang) in acupuncture combined with cognitive function training, and the differences after treatment obviously indicated that the acupuncture method was feasible and effective. According to the statistical analysis of the literature on acupuncture treatment of PD cognitive impairment in recent years, MMSE and MOCA were used as the criteria to determine the prognosis of the disease. Since this disease belongs to the category of dementia and is related to the level of education received, it should also be considered to include the level of education. Acupuncture and moxibustion in the treatment of cognitive impairment in PD were mostly combined with cognitive function training, but few were in the acupuncture treatment group alone, indicating that functional training was still the main way in clinical practice. Baihui and Sishencong were selected as the main acupoints for acupuncture, so as to achieve the purpose of awakening the mind, opening the oritions and filling the lean pulp, and to provide reference for clinical practice.

\subsection{Anxiety and Depression}

Affective disorders in Parkinson's disease are mainly manifested by anxiety and depression, and the incidence of which is about 35\%-50\% according to the survey [12]. Parkinson's disease with depression can lead to cognitive decline, quality of life decline, increased risk of suicide, and great harm to society and family. At present, the pathogenesis of this disease is still unclear, but it is generally believed to be caused by endogenous biological factors and exogenous psychological factors [13]. Praxol, Paxetine and other drugs are recommended in current guidelines [14]. In recent years, there has been a popular theory based on the brain-enteric-bacterial axis in the treatment of this disease by acupuncture and moxibustion. Some studies believe that intestinal flora can participate in the regulation of anxiety, depression and other symptoms, and intestinal flora can internally regulate patients' depression mood through acupuncture intervention [15-17]. Although the theory of intestinal flora is a new molecular mechanism for the treatment of this disease, the number of relevant articles is still insufficient, and the clinical treatment is based on the conventional meridians and acupoints. Xia Yi [18] used electroacupuncture combined with drugs to treat Parkinson's disease depression, and the effective rate was $90.0 \%$. After treatment, the score of Hamilton scale was significantly reduced. Wang Xiaomei [19] used heat-sensitive moxibustion as an adjuvant treatment for Parkinson's disease depression, and the effect was 95.0\%. Lei Jun [20] used bilateral Taichong, Fengfu and to treat this disease, with a significant curative effect of $80.9 \%$. According to the literature in recent years, acupuncture and moxibustion treatment of this disease mainly starts from liver, kidney and blood stasis. In addition, the literature on Heat sensitive moxibustion treatment of this disease [19] pointed out that the TCM syndrome has obvious limitations. Qi and blood failure is one of the most common syndesms of PD affective disorder, and Qi deficiency and blood stasis syndrome heart, spleen and blood deficiency are common causes of depression patients. Parkinson's disease patients with mood disorders are more likely to have the performance of Qi deficiency. Therefore, the treatment principle should be tonifying qi, promoting blood circulation and removing blood stasis, strengthening heart and spleen, warming and tonifying, promoting blood circulation and dredging collaterals. As a characteristic therapy of acupuncture and moxibustion, Heat sensitive moxibustion is a better warm tonic treatment program.

\section{Autonomic nervous dysfunction}

\subsection{Constipation}

PD constipation is a common high incidence of non-motor symptoms, and its incidence fluctuates within a wide range of $20 \%-89 \%$, mainly manifested as gastrointestinal disorders such as difficulty in defecation, reduced frequency, abdominal distension and discomfort, and decreased appetite [21-22]. At present, the main mechanisms of PD constipation include: human functional colonic transport delay, anorectal sphincter relaxation and intestinal flora imbalance; Side effects caused by external factors of anti-PD drugs; Other factors such as mental depression, lack of exercise and habit of long-term constipation in the elderly [23-25]. At present, the first drugs to be selected 
clinically are intestinal activators and osmotic laxatives as routine drugs for PD, but such drugs should not be used for a long time, otherwise dry stools and intestinal dysfunction will be aggravated [26]. Through clinical studies, acupuncture and moxibustion as an external treatment to intervene gastrointestinal function peristalsis is indeed effective, it can not only improve patients' constipation, but also reduce the dependence on drugs and reduce side effects. Jiang Lei [27] combined acupuncture and moxibustion treatment on the basis of conventional western medicine treatment, showing that acupuncture and moxibustion significantly improved patients' constipation, and the efficacy was better than western medicine treatment alone. Zhang Yu [28] used acupuncture to treat middle and late stage constipation to achieve general hysteresis and improve constipation symptoms. In recent years, acupuncture and moxibustion has been widely used in treating PD constipation, and the results are all effective. Studies have shown that the mechanism of acupuncture treatment for this disease is to achieve the therapeutic purpose by regulating the balance function of sympathetic and parasympathetic nerves [29]. According to literature review, it is found that the treatment of PD constipation is multi-choice: hand and foot Yangming meridian is used to recuperate the intestines and stomach, conduct stagnation and constipation, and then Tianshu, Qihai, warm acupuncture or mild moxibustion is used to promote the qi and energy, which can help the movement. Since PD constipation occurs most frequently in middle-aged and elderly people, and such people are mostly deficient in liver and kidney, and their conditions are long and coldheat complex, acupuncture combined with moxibustion and combination of manual reinforcing and reducing is the best treatment for this disease according to the literature.

\subsection{Urinary dysfunction}

PD bladder and urethral dysfunction is also known as neurogenic urination disorder because of the decreased control of the brain on urination related to neuropathy, which is usually caused by clinical manifestations such as frequent urination, urgent urination and dysuria [30]. The incidence of this disease in PD patients accounts for $40 \%-70 \%$, and it is also a common accompanying symptom of PD. At present, the traditional clinical medication is the anticholinergic drug tolterodine, but there are many side effects after medication, and the surgical treatment effect is not ideal [31-32]. On the basis of tolterodine combined with electroacupuncture, Chen Yiliang [33] observed the frequency of urination, frequency of urinary incontinence and volume of urine of the subjects, and the comparison of the treatment results before and after suggested that acupuncture combined with western medicine in the treatment of this disease effectively alleviated the situation of bladder activity and reduced urinary discharge and frequency. There are too few reported cases of clinical PD bladder dysfunction, and the sample size is not enough to support the efficacy of acupuncture and moxibustion in the treatment of this disease, so the persuasion is not strong. In addition, it is necessary to set up a simple acupuncture group to reduce interference factors to verify the efficacy.

\section{Difficulty swallowing}

As one of the most common complications of non-motor symptoms of PD, dysphagia occurs in the range of $18.5 \%-100 \%$, mainly manifested as eating difficulties, salivation at the mouth, food leakage, and choking easily, especially when eating liquid food, resulting in the risk of aspiration and further lung infection [34]. Research thinks that the pathogenesis of PD swallowing disorder with dopamine and the dopaminergic neural system are closely related: located in intramedullary basal ganglia of the dopamine in starting the swallow has a key role, PD patients lack of dopamine this substance, intramedullary nervous system startup dysphagia, oropharynx muscle stiffness with swallowing hard, eventually become the swallowing disorder [35]. Levodopa is a commonly used drug in the treatment of this disease, and its efficacy is still controversial. Studies have found that acupuncture can increase dopamine content in the brain and stimulate dopamine neurons, thus promoting the recovery of swallowing function [36]. Zhao Yao [37] showed that the total effective rate of $83.3 \%$ in the treatment group was significantly better than $50 \%$ in the control group in the treatment of PD dysphagia by warm acupuncture combined with functional training. Wang Hongyan [38] acupuncture combined with oral training has a definite effect on PD patients with dysphagia, which significantly improves the living ability of patients and reduces the related side effects caused by drugs. PD deglutition disorders and post-stroke deglutition disorders are hot topics in recent years. However, acupuncture and acupoints selected for PD deglutition disorders are all stimulated in the neck, tongue, pharynx and their surrounding areas, which are all located in the glossopharynx, the sensory ligand area of the vagus nerve and the local muscle structure of glossopharynx. Acupuncture at the point of the neck can radiate the sense of needles along the tongue to the tongue base and even the throat, so as to excite the superior center, and then pass down to the glossopharyngeal muscles to restore the function of autonomous movement of local muscles. At present, although the medical community's attention to PD swallowing shows an increasing trend year by year, a large number of clinical studies and experimental data are still needed to demonstrate the exact efficacy of acu- 
puncture on this disease.

\section{Sleep and wakefulness disorders}

Insomnia is the most common sleep disorder in PD and often appears before motor symptoms. PD patients usually do not have difficulty falling asleep, but maintaining sleep is very challenging for many patients, which is called fragmented sleep and also known as sleep maintenance insomnia, with up to $80 \%$ of patients reporting sleep deprivation and early morning wakefulness [39]. Loss of dopaminergic neurons in PD's substantia nigra is an important pathological basis for insomnia in Parkinson's patients [40]. Benzodiazepines, represented by clonazepam, are often used to alleviate general insomnia symptoms, and are also used as the chosen medication for the treatment of PD insomnia. It has been reported that $90 \%$ of patients with REM sleep behavior disorder symptoms are partially or completely relieved [41]. Yu Zhenhua [42] observed the clinical efficacy of Rehmannia glutinosa (Rehmannia glutinosa) combined with acupuncture in patients with Parkinson's insomnia, which played an obvious effect of tranquilizing the mind and tranquilizing the mind. Huang Na [43] used Fang's head needle, selected Fu Xiang head acupoint area, signal acupoint area, thinking acupoint area and other combined with Medoba for treatment, to improve autonomic nerve functional activity and improve insomnia symptoms. Li Lin [40] combined traditional Chinese medicine and acupuncture to improve the sleep quality of PD patients from the perspective of restraining abnormal brain excitement and balancing brain function. Yang Shihong [44] added body acupuncture treatment on the basis of Medoba, which not only improved patients' sleep disorders, but also reduced nausea, constipation, insomnia, iso switch and end-of-dose phenomenon, delaying the occurrence time of end-of-dose phenomenon. At present, acupuncture is used to treat PD sleep disorder, and body acupuncture is the most common treatment and often combined with medication. The researchers selected Guipi Decoction to treat this disease from the demonstration point of deficiency of heart and spleen. Acupuncture alone is rarely used to treat PD sleep disorders clinically, and there are few literatures. After reviewing the literature, it was found that the treatment of this disease with Fang's head acupuncture was mostly mentioned in relevant literature, but the number was relatively small. It is still necessary to further expand the sample size and extend the observation duration.

\section{Sensory disorders}

At present, the research on PD pain-related diseases in China is still in an incomplete stage, so there is no unified definition and effective treatment for PD pain, and the existing treatment methods mostly continue with drugs and surgery [45]. PD-related pain accounts for a high proportion in patients, and 40\%-85\% May suffer from painful sensory disorders [46]. Studies have found that there are various types of PD pain with different pathogenesis, among which the most common occurrence rate of skeletal muscle pain is higher than $70 \%$, which is mainly caused by the muscle stiffness and abnormal posture of PD itself, and the inflammatory substances formed by muscle pulling and tearing injuries during activities are closely related [47]. Li Xiaoyuan [48] summarized the experience in the treatment of PD with fire needles, and achieved the effect of warming Yang and relieving pain according to the action characteristics of fire needles to warm meridians and channels. Yu Nannan [49] used floating needles to treat limb pain associated with Parkinson's disease, rapidly alleviating pathological muscle tension in a short time, and thus achieving the purpose of pain treatment. Pain, as the dominant disease in the treatment of acupuncture and moxibustion, can relieve pain through stimulation according to the theory of local or distal collaterals dredging. Currently, although there have been reports on PD sensory disorder in the literature, the number is few and there is lack of sufficient data demonstration. The clinical treatment is still dominated by western medicine or surgical operation.

\section{Conclusions and Prospects}

Parkinson's disease is a refractory disease with various manifestations and no fixed pattern according to the patient's constitution [50]. Studies have shown that clinical use of anti-Parkinson drugs may lead to new neurotransmitter dysfunction, such as mental symptoms, gastrointestinal discomfort, cardiovascular symptoms, etc. [51], which leads to very limited drug therapy. Therefore, it is very important to choose the treatment with low side effects for PD patients. Through the above literature review and analysis, it was found that acupuncture treatment of PD-NMS symptoms significantly improved, improved the quality of life of patients and reduced the occurrence of side effects. However, it should be noted that most clinical observations rarely have a "single acupuncture treatment group", so it is not convincing for the mechanism of action of acupuncture. Therefore, in the future clinical studies, further studies should be conducted to expand the sample size and set up a separate acupuncture treatment group, so 
as to provide strong data support and theoretical evidence for the treatment of this disease by acupuncture and moxibustion.

\section{References}

[1] Ma, C. L., Su, L., Xie, J., et al. (2014). The prevalence and incidence of Parkinson's disease in China: A systematic review of analvsis [J]. J Neural Transm (Vienna), 2014, 121(2): 123-134.

[2] Li Mengzhu, Huang Shijing. (2018). Treatment of Parkinson's disease with qi deficiency and blood stasis [J]. Research of Traditional Chinese Medicine, 2018, 31(2): 8-10.

[3] Shen Jikang, Ye Min, Liu Weiguo, et al. (2010). Clinical Neurology, 2010, 23(04): 251-253. (in Chinese with English abstract)

[4] Sun Miaoxuan, Zhang Xiong. (2013). Journal of Shanghai University of Traditional Chinese Medicine, 2013, (5): 41-48, 69. (in Chinese)

[5] Fox, S. H., Katzenschlager, R., Lim, S. Y. (2018). International Parkinson and Movement Disorder Society Evidence-Based Medicine Review: update on treatments for the motor symptoms of Parkinson's disease. Mov Disord., 2018; 33(8): 1248-1266.

[6] Bueters, T., von Euler, M. Bendel, et a1. (2008). Degeneration of newly formed CAl neurons following global phenomena in the rat [J]. Exp Neurol, 2008, 209(1): 114-124.

[7] Klaus Seppi, K. Ray Chaudhuri. (2019). Update on treatments for nonmotor symptoms of Parkinson's disease-an evidence-based medicine review. 2019 Feb; 34(2): 180-198.

[8] Cao Liangsong, Li Xiujuan, Jiang Guangyao, et al. (2018). Effects of acupuncture and moxibustion combined with cognitive function training on cognitive function and motor function in patients with Parkinson's disease [J]. Clinical Journal of Acupuncture and Moxibustion, 2018, 34(04): 9-12.

[9] Zhang Lijuan. (2016). Clinical Rational Drug Use, 2016, 9(25): 80-81. (in Chinese with English abstract)

[10] Zhang Xiaoli, Feng Weiju, Chen Yiliang, et al. (2013). Shanghai Journal of Acupuncture and Moxibustion, 2013, 32(04): 263-265.

[11] Ye Jiasheng. (2016). Shanghai Journal of Acupuncture and Moxibustion, 2016, 35(09): 1053-1055. (in Chinese)

[12] Li, Y. M., Shi, J., Li, T, (2018). Characterization and Characterization of TCM syndrome in patients with Parkinson's disease. Journal of Clinical Pharmacology, 2018, 21(1): 73-84, 11(1): 44-47.

[13] Yuan, Q. J. (2014). Therapeutic Observation on Differentiation Treatment with Tonifying-kidney, Harmonizing-liver and Resolving-depression for Parkinson's Disease with depression. Master Thesis of Beijing University of Chinese Medicine, 2014.

[14] Schrag, A., Barone, P., Brown, R. G., et al. (2007). Depression rating scales in Parkinson’s disease: critique and recommendations [J]. Mov Disord., 2007, 22(8): 1077-1092.

[15] Xie Jiageng, Wang Shun, Liang Yao. (2016). Clinical study of acupuncture and moxibustion combined with bifidobacterium triad in the treatment of Parkinson's depression [J]. World Journal of Traditional Chinese Medicine, 2016, 11(10): 2116-2119, 93, (02): 37-38.

[16] Li Yuan, Wang Shun, Bai Yan, et al. (2018). Clinical Journal of Acupuncture and Moxibustion, 2018, 34(10): 79-82. (in Chinese with English abstract)

[17] Cai Ying, Yu Pengyang. (2020). The value of acupuncture and moxibustion in the treatment of Parkinson's disease depression [J]. Clinical Research, 2020, 06-0062-01.

[18] Xia Yi, Wang Haidong, Ding Ying, et al. (2012). Effect of electroacupuncture and drug therapy on serum BDNF in patients with Parkinson's disease [J]. Chinese Acupuncture and Moxibustion, 2012, 32(12): 1071-1074.

[19] Wang Xiaomei, Rong Chunjiao, Zhang Fuqing. (2020). Clinical efficacy and mechanism of thermosensitive moxibustion in adjuvant treatment of Parkinson's disease depression [J]. 2020, 18(1): 33-39. (in Chinese)

[20] Mr Leven. (2012). Clinical observation on the treatment of Parkinson's disease depression with acupuncture and moxibustion [J]. Seek Medicine (second half month), 2012, 10(04): 76-77.

[21] Zhang, J., Zhang, J., Zhang, J., et al. Characterization of Gastrointestinal glucomatosis in adult adult adult [J]. Advances in Bioscience and Bioscience, 2007, 19(2): 187-194.

[22] Zhang Li. (2014). Clinical study on the effect of anti-shock zhospasmodic capsule on constipation in Parkinson's disease with blood stasis and wind-motion [D]. Anhui: Anhui University of Chinese Medicine, 2014.

[23] Zeng Qi, Liu Zhibin, Wang Yuan, et al. (2020). Journal of Liaoning University of Traditional Chinese Medicine, 2020, 22(08): 40-44. (in Chinese)

[24] Streit, W. J., Xue, Q. S. (2015). Dementia with Lewy bodies in Microglia [J]. Brain Behav Immun, 2015, 10 (55): $191-201$.

[25] Zhang, H., Gu, Z., An. J., et al. (2014). Non-motor symptoms in treated and untreated Chinese patients with early Parkinson's [J]. Chin J Med., 2014, 5(232): 129-136. (in Chinese)

[26] Song Zhijian. (2019). Progress in the treatment of constipation symptoms in Parkinson's disease by traditional Chinese and 
western medicine [J]. World Newest Medical Information Abstracts, 2019, 19(66): 86-87.

[27] Lei Jiang, Shangsheng Ke, Jing Jin. (2020). Clinical observation of acupuncture and moxibustion for functional constipation in Parkinson's disease [J]. Neurological Injure and Functional Rebuilding, 2020, 15(12): 749-750. (in Chinese)

[28] Zhang Yu, Wei Yan. (2020). Clinical effect analysis of acupuncture and moxibustion in treatment of constipation in middle and advanced Parkinson's disease [J]. Electronic Journal of Clinical Medical Literature, 2020, 7(05): 49.

[29] Sun Lianzhu, Ma Ruijie. (2011). Neurology and Neurorehabilitation, 2011, 8(03): 158-160. (in Chinese with English abstract)

[30] Wingea, K., Fribergb, L., Werdelin, L., et al. (2005). Relationship between nigrostriatal dopaminergic degeneration, urinary symptoms and bladder control in Parkinson's disease [J]. European Journal of Neurology, 2005, 12: 842-850.

[31] Sakakibara, R., Shinotoh, H., Uchiyama, T., et al. (2001). A questionnaire-based as-sessment regimen of organ dysfunction in patients with Parkinson's disease [J]. Auton Neo-sci, 2001, 51(4): 284-294; 92(1-2): 76-85.

[32] Chaudhuri, K. R., Martinez-Martin, P., Brown, R. G., et al. (2007. The metricpropertise of a novel non-motor symplomsscale for Parkinson's disease: Results from an international pilot study [J]. Mov Disord., 2007, 22(13): 1901-1911.

[33] Chen Yiliang, Feng Weiju, Zhang Xiaoli, et al. (2012). Treatment of Parkinson's disease complicated with bladder hyperactivity with acupuncture and medicine [J]. China Acupuncture and Moxibustion, 2012, 32(03): 215-218.

[34] Wood, L. D., Neumiller, J. J., Setler, S. M. (2010). Analgesia and nonmotor symptoms of Parkinson's Disease: a meta-analysis [J]. AM J Geriatr Pharmacother, 2010. 8: 294-315.

[35] Volonte, M. A., Porta, M., Comi, G. Neurol Sci., 2002, 23(Suppl 2): S121.

[36] Sun Lianzhu, Ma Ruijie. (2011). Journal of Neurology and Neurorehabilitation, 2011, 3: 158.

[37] Zhao Yao, Liu Ping, Yin Yanying, et al. (2015). Effect of acupuncture and moxibustion combined with functional training on deglutition dysfunction in Parkinson's disease [J]. Modern Distance Education of Traditional Chinese Medicine in China, 2015, 13(07): 61-63.

[38] Wang Hongyan, Zuo Guanchao, Liu Jing, et al. (2020). Effect of acupuncture combined with oral sensorimotor training on deglutition disorder in Parkinson's disease [J]. Shanghai Journal of Acupuncture and Moxibustion, 2020, 39(04): $440-445$.

[39] Videnovic, A. (2017). Management of sleep disorders in Parkinson’s disease and multiple system atrophy. Mov Disord., 2017 May; 32(5): 659-668.

[40] Lilin. (2018). Guangming Traditional Chinese Medicine, 2018, 33(14): 2043-2044. (in Chinese with English abstract)

[41] Howell, M. J., Arneson, P. A., Schenck. C. H. (2011). A Novel Therapy for REM Sleep Behavior Disorder (RBD) [J]. J Clin Sleep Med., 2011, 7(6): 639-644a.

[42] Yu Zhenhua. (2019). Clinical observation of dihuang yinzi plus subtraction combined with acupuncture in the treatment of patients with Parkinson’s disease [J]. China Medical Guide, 2019, 17(25): 7-8.

[43] Huang Na, Huang Linna, An Junming. (2014). Clinical effect of Fang's head acupuncture on insomnia in Parkinson's disease [J]. Shaanxi Journal of Traditional Chinese Medicine, 2014, 35(3): 348-349.

[44] Yang Shihong. (2014). Clinical efficacy and adverse reactions of metobar combined with acupuncture in the treatment of Parkinson's disease [J]. Modern Medicine and Health, 2014, 30(17): 2692.2693.

[45] Lin Xiaoguang, Liu Weiguo, Lin Xingjian. (2014). Clinical Neurology, 2014, 27(4): 244-247. (in Chinese with English abstract)

[46] Li Yao, Zu Jie, DoNG Liguo, et al. (2019). Clinical characteristics and related factors of Parkinson's disease with pain [J]. Chinese Journal of Neuroimmunology and Neurology, 2019, 26(04): 265-268+280.

[47] Katunina, E., Titova, N. (2017). The epidemiology of nonmotor symptoms in Parkinson's disease (Cohort and Other Studies) [J]. Inter Rev Neurobiol., 2017: 91-110.

[48] Li Xiaoyuan, Wang Hongjuan. (2017). A case study of fire acupuncture in the treatment of Parkinson's disease pain [C]. Proceedings of 2017 World Acupuncture and Moxibustion Academic Conference and 2017 Annual Meeting of Chinese Acupuncture and Moxibustion Society. Chinese Society of Acupuncture and Moxibustion, 2017: 340-344.

[49] Yu Nannan, Ma Lin, Bi Haiyang, et al. (2020). Clinical study of floating needle in treatment of limb pain in Parkinson's disease [J]. Clinical Journal of Acupuncture and Moxibustion, 2020, 36(01): 37-40.

[50] Kawasaki, M., Kawasaki, M., Kawasaki, M., et al. (2012). Parkinsonism in Parkinson’s disease: a review [J]. J Clin Pathol, 2012, 35(1): 87-91.

[51] Tong Qijin. (1993). The side effects of seven drugs against Parkinson's disease [J]. Chinese Journal of Hospital Pharmacy, 1993, (02): 37-38. 